\title{
$\checkmark$ Research Square \\ Excitation of Surface Plasmon-Polariton Wave at Both Interfaces of a Silver Thin Film in Two-Layer Kretschmann geometry
}

Ferydon Babaei ( $\square$ fbabaei@qom.ac.ir)

University of Qom https://orcid.org/0000-0003-1704-0474

Seyed Abas Seyyedi

University of Qom

\section{Research Article}

Keywords: surface plasmon- polariton, oblique angle deposition, columnar thin film

Posted Date: May 6th, 2021

DOI: https://doi.org/10.21203/rs.3.rs-475773/v1

License: (c) (i) This work is licensed under a Creative Commons Attribution 4.0 International License.

Read Full License 


\title{
Excitation of surface plasmon-polariton wave at both interfaces of a silver thin film in two-layer Kretschmann geometry
}

\author{
Ferydon Babaei ${ }^{\text {a, }}{ }^{*}$, Seyed Abas Seyyedi ${ }^{\text {a }}$ \\ a) Department of Physics, University of Qom, Qom, Iran \\ *Corresponding Author: \\ Ferydon Babaei, Email: fbabaei@qom.ac.ir
}

\begin{abstract}
In this work, a $\mathrm{TiO}_{2}-\mathrm{Ag}$ composite structure was fabricated by combination of physical vapor deposition and oblique angle deposition. The nanostructure of the $\mathrm{TiO}_{2}$ columnar thin film was analyzed using the field emission scanning electron microscopy. The excitation of the surface plasmon-polariton waves was studied using an optical setup that is called the two-layer Kretschman geometry. The results showed that two surface plasmon-polariton modes are propagated for $\mathrm{TiO}_{2}$ dielectric thin film at oblique deposition that they are related to metal-air and $\mathrm{TiO}_{2}$ - metal interfaces. The surface plasmon-polariton modes were more localized on interfaces at glancing angles of incident flux vapor. In normal incident of flux vapor only one surface plasmon-polariton mode can be excited at interface of metal-air. The results of this research may be applied in simultaneous sensing of the different molecules, chemical species and nanoparticles with different sizes.
\end{abstract}

Keywords: surface plasmon- polariton, oblique angle deposition, columnar thin film

\section{Introduction}

One nanotechnology fields is the study of the interaction of light with metal nanostructures, currently known as plasmonics. Surface plasmon resonance (SPR) takes place when the incident light frequency equals the frequency of the oscillations of the density of the conducting electrons. By applying an electromagnetic wave, one can excite surface plasmon wave at the metal-dielectric 
interface. The electric field of a surface plasmon wave exponentially decays perpendicular to the surface of the interface, able to be amplified by the incident of an electromagnetic wave. The excitation of surface plasmons with an electromagnetic wave with linear polarization is called surface plasmon-polariton(SPP) [1]. In quantum viewpoint, SPPs are quasi-particles resulting from the coupling of plasmons in metals to polarized photons of light. But, the trains of SPPs can be viewed classically as SPP wave at metal-dielectric interface [2]. SPPs are very sensitive to environmental conditions and properties, and this property can be used in sensing different types of adsorbed nanoparticles and chemical species [3].

Surface plasmons are not directly excited by incident photons in the planar dielectric medium as the dielectric medium does not have enough free electrons for coupling with the incoming photon and excitation of the surface plasmons. In this case, the parallel component to interface of incident photon wave vector is not equal to the real part of the SPP wave vector. Because the momentum of the incident photons is smaller than the momentum of the surface plasmons, and the photon cannot be coupled to the surface plasmon [4]. Hence, those configurations must be used to compensate for the lack of momentum, and the most common experimental method for detection of surface plasmons is Kretschmann configuration, which is a structure, composed of metal, dielectric, and prism [5]. In this configuration, the incident beam passes through a prism and reaches the metal surface. At angles greater than the critical angle (total internal reflection phenomena), the photon can be coupled to surface plasmons and excited them. By measuring the intensity of reflected light, a minimum sharp is observed in the spectra, showing SPP excitation [6].

First, the focus of the researchers on SPP excitation was on the metal-isotropic dielectric interface. With the emergence of the new generation of thin films called sculptured thin films (STFs), the studies on SPP excitation at the interface of metal and anisotropic dielectric has increased [7-14]. STFs are a set of parallel columns made by combination of an oblique angle deposition (OAD) and physical vapor deposition(PVD). STFs have columns with a diameter of 50 to $100 \mathrm{~nm}$, each of which has clusters with a diameter of 3 to $5 \mathrm{~nm}$ [15]. The direction of the columns growth during 
coating by stepper motors can change continuously or abruptly, resulting in the growth of a thin layer with curved nano columns.

The study intends to fabricate a structure composed of columnar titanium dioxide thin film, silver thin film with a combination of OAD and PVD, which the columnar thin film is a simple form of STFs. The column morphology of titanium dioxide was analyzed by field emission scanning electron microscopy (FESEM). The excitation of SPP was confirmed with an optical setup called two-layer Kretschman geometry. In Kretschman geometry, SPP can be excited only at the external interface of the metal. In two-layer Kretschman geometry, an additional dielectric should be deposited with a refractive index less than a prism between the prism and the metal film [1]. In this case, SPP can be excited at internal interface of the metal. In Section 2, the experiment method is presented, followed by the results and analyses in Section 3.

\section{Experiment method}

Silver and titanium dioxide raw materials were prepared as tablets with $99 \%$ purity. The microscope slide substrates were cleaned using acetone and alcohol in ultrasonic bath. They were placed on a steel substrate holder in the shape of a circle with a diameter of $8 \mathrm{~cm}$. Then the titanium dioxide $\left(\mathrm{TiO}_{2}\right)$ tablets were deposited on the microscope slide using OAD and PVD. The base pressure inside the vacuum chamber was $5 \times 10^{-6}$ torr, which reached $10^{-5}$ torr during evaporation. The thickness of thin film was controlled by a quartz crystal monitor. Three samples of $\mathrm{TiO}_{2}$ with about thickness $700 \mathrm{~nm}$ were coated at deposition angles of $0^{\circ}, 75^{\circ}$ and $85^{\circ}$ with evaporation rate $0.5 A^{\circ} / S$ (Fig. 1).The distance from the source to the substrate was $60 \mathrm{~cm}$. Then 40 to $50 \mathrm{~nm}$ silver was deposited on $\mathrm{TiO}_{2}$ by $\operatorname{PVD}\left(\alpha=0^{\circ}\right)$.

The samples from the microscopic slide side by immersion oil $(n \approx 1.52)$ were glued to the base of a right triangular prism (BK7-glass) with a refractive index of 1.52. A helium-neon laser with a power of $12 \mathrm{~mW}$ operating at $\lambda \approx 633 \mathrm{~nm}$ was used as a source of monochromatic, collimated light. Also, our set up had two polarizers and an optical calibrated turntable(Fig.2). The first polarizer had a role 
in adjust incident light intensity and the second polarizer controlled the type of polarization of the incident light (P or $\mathrm{S})$.

Minimum deviation method was used to ensure the refractive index of the prism, where the average value $n=1.5105$ was obtained after measuring 4 times. In all the samples placed on the prism the optical calibrated turntable was rotated from $\phi=-60^{\circ}$ to $\phi=+40^{\circ}$ with increment one degree and the intensity of the reflected light was measured by a multimeter. The $\phi$ was the angle between the incident beam and the perpendicular line on the lateral point of the prism, whose relation with the internal angle $\theta$ is obtained from $\theta=\frac{\pi}{4}-\operatorname{Arcsin}\left(\frac{\sin \phi}{n}\right)$ relation using Snell law (Fig.3).

\section{Results and analyses}

Fig.4 shows the FESEM electron micrographs of the surface and cross section of $\mathrm{TiO}_{2}$ columnar thin film deposited on the microscope slide at $\alpha=0^{\circ}$. It is clear from the Fig.4 that the columns have grown vertically relative to the substrate surface $\left(\beta=0^{\circ}\right)$ and thin film have less porosity. In published researches, it has been shown experimentally and theoretically that when the direction of the incident vapor flux is close to the substrate surface, the columns will grow slightly inclined perpendicular line to the substrate surface and the thin film will have more porosity. The relationship between deposition angle and rise angle of the columns at the incident vapor fluxes close to the substrate surface is obtained from Tait equation $\beta=\alpha-\operatorname{Arcsin}\left(\frac{1-\cos a}{2}\right)$ [16]. However, it follows empirical tangent rule $\beta=\frac{1}{m} \tan \alpha$ for almost vertical incident of vapor flux, where $\mathrm{m}$ is close to 2 and depends on the type of evaporating material [17]. It is obvious from Fig.4 that our work is consistent with others literature in vertical incident of vapor flux that the columns should have grown vertically [7].

FESEM electron micrographs of the surface $\mathrm{TiO}_{2}$ columnar thin film deposited on the microscope slide at $\alpha=75^{\circ}$ are given in Fig.5. If the vapor flux beams reach at a greater angle relative to the perpendicular line on substrate surface, we expect more inclined nano columns and more porosity. 
Tait equation predicts the columns with $\beta=53^{\circ}$. Fig.5 shows nano columns with a rise angle about $\beta=60^{\circ}$, differing $13 \%$ from the value obtained from the Tait equation. However, up to $20 \%$ of the error is normal because of the involvement of many parameters in deposition: parameters such as evaporation material, angular distribution of flux, flux particle energy, substrate surface roughness and most importantly substrate temperature play a role in the formation of nano columns and porosity space between columns. The length of the columns naturally increases with increase in the thickness and orientation of columns in the direction of atomic vapor flux. Nonetheless, the effect of their shadowing increases as the length of the columns increases, the probability of the deposition of atomic particles on the columns has increased and the filling of void spaces becomes much less. The density of thin film is inversely related to their porosity. This means that the larger the rise angle of the nano columns $\beta$, the greater the atomic shadowing effect will be, resulting in greater porosity. Higher porosity results in a lower density thin film. The diameter of nano columns increases with the coating time at larger $\alpha$ angles, because of the more inclination of the nano columns, the probability of particle deposition on the surface of the columns increases and the continuation of the coating and increase in temperature, and thus the effect of atomic diffusion on the tops of the nano columns, causes their diameter to broaden so that the cross section of the columns will be elliptic.

The FESEM electron micrographs of the surface $\mathrm{TiO}_{2}$ columnar thin film at $\alpha=85^{\circ}$ are shown in Fig.6. It is observed that the nano columns formed in this case are more porous than in Fig.5 and the rise angle of columns was around $\beta=67^{\circ}$.This value had to be $\beta=58^{\circ}$ from Tait equation, showing a difference of about $15 \%$. The boarding of the columns is seen in this case too.

The three captured snapshots for a silver thin film deposited on microscope slide for S polarization are shown in Fig.7. It clarifies that the blurring is not seen in the intensity of the reflection spectra, and thus for this type of polarization, the surface plasmons are not excited due to the perpendicularity of the electric field to the incident plane (a plane contains a vertical line to the substrate surface and the incident light wave vector). The captured snapshots for $\mathrm{P}$ polarization are reported in Fig.8.The middle slide shows a blur in the intensity of the reflection spectra and the corresponding angle read 
from the optical calibrated turntable is the angle where the energy from photons to surface plasmons is transferred and the surface plasmon resonance is occurred.

In Fig.9 the measured reflection spectra in terms of angle $\theta$ for both polarizations $P$ and $S$ is shown for a composite structure of $40 \mathrm{~nm}$ silver thin film and $500 \mathrm{~nm} \mathrm{TiO}_{2}$ columnar thin film at deposition angle $\alpha=85^{\circ}$. It is seen that two valleys have emerged for $\mathrm{P}$ polarization at angles $\theta=46^{\circ}$ and $\theta=62^{\circ}$.The smaller $\theta_{\mathrm{SPP}}$ is devoted to excitation of SPP at metal-air interface (external interface), and the other valley, occurring at a larger angle, is related to the SPP excitation at $\mathrm{TiO}_{2}-\mathrm{Ag}$ interface (internal interface). However, these two valleys are weakly seen in S polarization, which is because of anisotropy and birefringence in the $\mathrm{TiO}_{2}$ columnar thin film. The results obtained here are consistent with those of others where SPP can also be excited at internal interface of metal when a dielectric with a lower density than the prism is placed between the prism and the metal [1].The measured reflection spectra at $\alpha=75^{\circ}$ is given in Fig.10. It is observed that two valleys are appeared for $\mathrm{P}$ polarization at angles $\theta=31^{\circ}$ and $\theta=70^{\circ}$. The smaller angle relates to silver-air interface and the larger to $\mathrm{TiO}_{2}$-silver interface. The comparison of Figs. 9 and 10 shows that the valleys are wider at $\alpha=75^{\circ}$. The broadening of the valleys is because of the increase in damping of SPP waves and reduces the SPP propagation length. Additionally, as the valley is deeper and the smaller FWHM (full width in half minimum) of the valley, it shows that the SPP wave is more localized at interfaces, and the propagation length of the surface plasmon-polariton wave increases.

Fig.11 shows the measured reflection spectra from $\mathrm{TiO}_{2}-\mathrm{Ag}$ composite structure as a function of $\theta$ for both linear polarizations at $\alpha=0^{\circ}$.It clearly shows that only a SPP wave is seen at metal-air interface for $\mathrm{P}$ polarization. However, we did not expect this to be seen in $\mathrm{S}$ polarization. It is possible that during the deposition of the columnar thin film, the vapor flux does not strike vertically on the substrate surface in some places, and consequently we will have birefringence effect in $\mathrm{TiO}_{2}$ columnar thin film and also thin film had low void spaces between columns (see Fig.4). Thus, we will have the excitation of SPP for S polarization as Fig.11 shows. Moreover, the reflection spectra widening in the last layer is more than the previous layers, which is because of the non-localization of the SPP wave and its damper. 


\section{Conclusions}

A $\mathrm{TiO}_{2}-\mathrm{Ag}$ composite structure is fabricated by combination of physical vapor deposition and oblique angle deposition. In our work, the silver thin film is deposited by physical vapor deposition and $\mathrm{TiO}_{2}$ columnar thin film is prepared at deposition angles 0,75 and 85 degrees. The plasmonic characteristics of composite structure are studied by the optical setup called two-layer Kretschman geometry. The obtained results showed that two SPP waves can be excited in composite structure for both liner polarizations at glancing angles of incident vapor flux for $\mathrm{TiO}_{2}$ columnar thin film. The small angle of SPP wave is devoted to propagation of SPP at external interface of metal(metal-air) and the large angle is related to the SPP propagation at internal interface of metal $\left(\mathrm{TiO}_{2}-\mathrm{Ag}\right.$ interface $)$. It was found that only one SPP mode can be excited at external interface of a metal in composite structure when $\mathrm{TiO}_{2}$ is deposited at lower deposition angles.

\section{Acknowledgement}

This work was carried out with the support of the University of Qom.

This article is original, has been written by the stated authors who are all aware of its content and approve its submission, has not been published previously, it is not under consideration for publication elsewhere. The authors declare that they have no known competing financial interests or personal relationships that could have appeared to influence the work reported in this paper. We have not the Competing Interests in manuscript. F. Babaei proposed the idea of the project, performed the prepared the main manuscript text and figures. F. Babaei and S.A. Seyyedi did the optical part of work and also samples fabricated by S.A. Seyyedi.

\section{Funding}

"Not applicable".

\section{Availability of data and materials}

" Not applicable". 


\section{References}

[1] A. V. Zayats, I. Smolyaninov, and A. Maradudin, Nano-optics of surface plasmon polaritons, Phys. Rep 408 (2005) 131.

[2] M. Faryad, and A. Lakhtakia, Surface plasmon-polariton wave propagation guided by a metal slab in a sculptured nematic thin film,J.Opt 12 (2010)085102.

[3] J. A. Polo, T. G. Mackay, and A. Lakhtakia, Mapping multiple surface-plasmon-polariton-wave modes at the interface of a metal and a chiral sculptured thin film, J.Opt. Soc. Am. B 28(2011) 2656. [4] J. A. Polo, and A. Lakhtakia, On the surface plasmon polariton wave at the planar interface of a metal and a chiral sculptured thin film, Proc. R. Soc. A 456 (2009)87.

[5] A. Lakhtakia, Surface-plasmon wave at the planar interface of a metal film and a structurally chiral medium, Opt. Commun 279(2007)291.

[6] M. Mansuripur,A. R. Zakharian, and J. V. Moloney, Surface plasmon polaritons on metallic surfaces, Opt. Photon. News 18(2007) 44.

[7] J. A. Polo, T. G. Makay, and A. Lakhtakia, "Electromagnetic Surface Waves: A Modern Perspective", Elsevier, Waltham, USA(2013).

[8] F.Babaei, and M. Omidi, Characteristics of plasmonic at a metal /chiral sculptured thin film interface ,Plasmonics 8(2013)1051.

[9] F. Babaei, and S. Shafiian -Barzoki, Surface plasmon polariton propagation at the interface of a metal and an ambichiral nanostructured medium ,Plasmonics 9(2014)595.

[10] F.Babaei, and H. Savaloni, Optical absorption transitions in Mn star-like helical sculptured thin films, Plasmonics 13(2018)203.

[11] F. Babaei, and S. Shafiian -Barzoki, Surface plasmon-polariton modes at interface of a metal and an ambichiral sculptured thin film ,Plasmonics 9(2014)1481.

[12] S.H. Hosseininezhad, and F Babaei, Excitation of multiple surface plasmon-polaritons by a metal layer inserted in an equichiral sculptured thin film, Plasmonics 13(2018)1867.

[13] M. Fakharpour, F Babaei,and H Savaloni, Engineering Mn as tetragonal-like helical sculptured thin film for broadband absorption, Plasmonics 11(2016)1579.

[14] S.H. Hosseininezhad, and F Babaei, On the Propagation of Multiple Surface Plasmon-Polariton Waves by a Triangle Helix Thin Film in Sarid Configuration, Plasmonics 14(2019)1843.

[15] K. Robbie, J. C. Sit, and M. J. Brett, Advanced techniques for glancing angle deposition, J.Vac.Sci.Technol.B 16(1998) 1115.

[16] M. W. Horn, M. D. Pickett, R. Messier, and A. Lakhtakia, Blending of nanoscale and microscale in uniform large-area sculptured thin-film architectures, Nanotechnology 15 (2004)303.

[17] R. Messier, V. C. Venugopal, and P. D. Sunal, Origin and evolution of sculptured thin films, J.Vac.Sci.Technol.A 18(2000) 1538. 


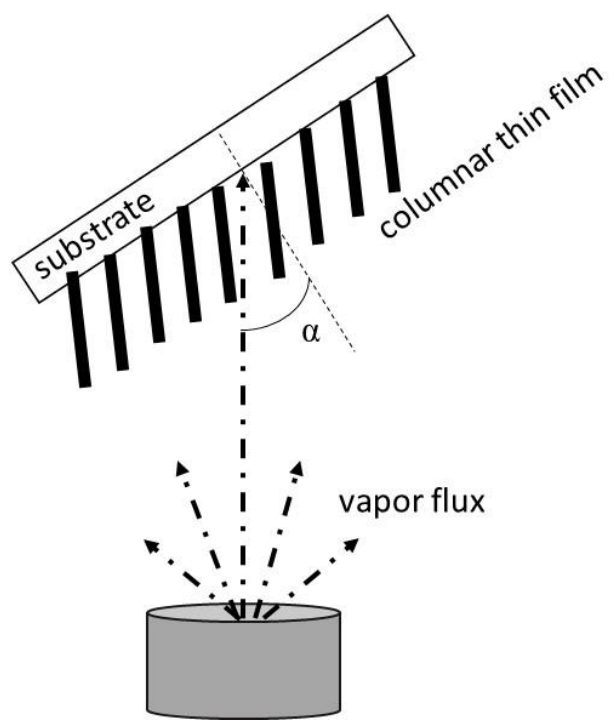

Fig.1.An illustration of oblique angle deposition(OAD) for growth of columnar thin film. The $\alpha$ is deposition angle.

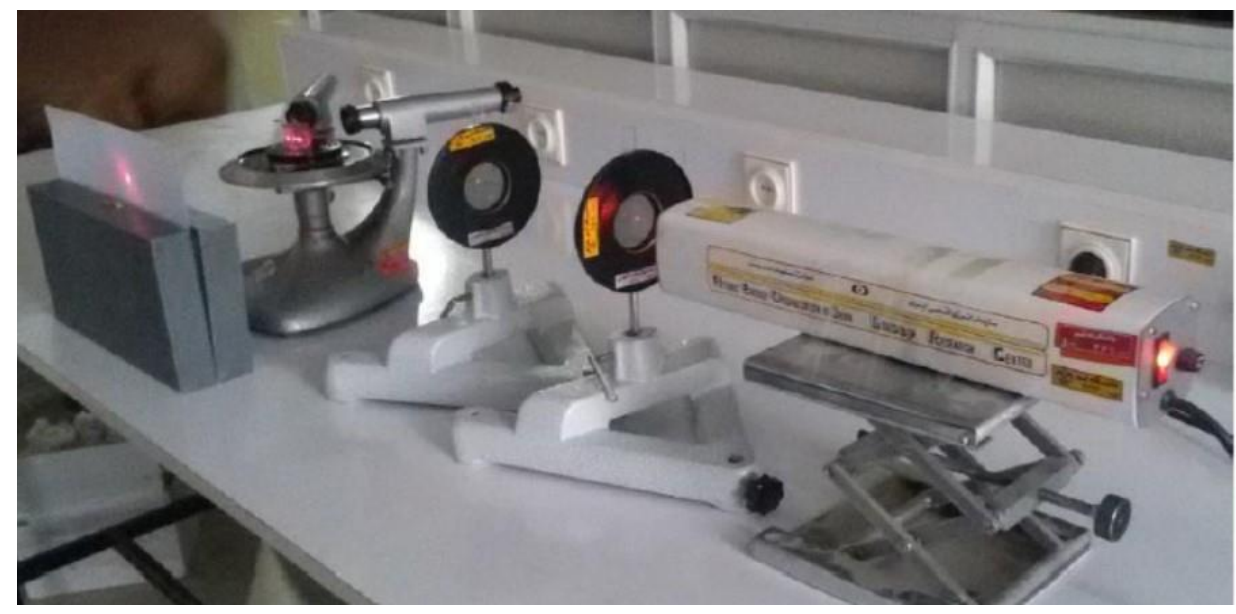

Fig.2.The optical setup for excitation of SPP. 


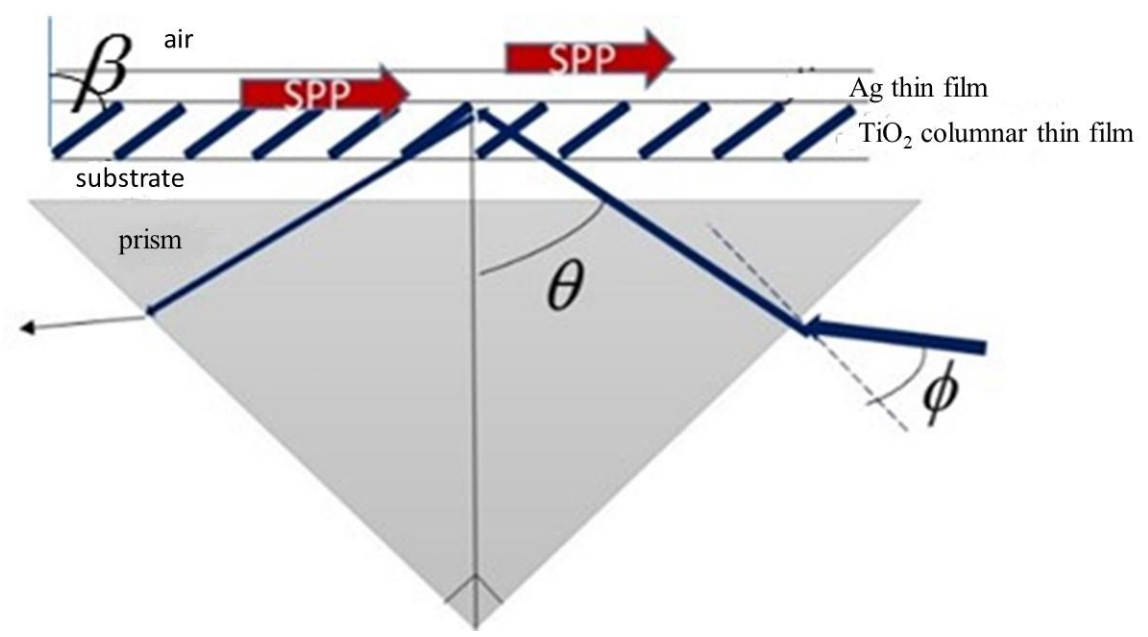

Fig.3.Shematic of two-layer Kretschmann geometry. The $\beta$ is rise angle of columnar thin film.
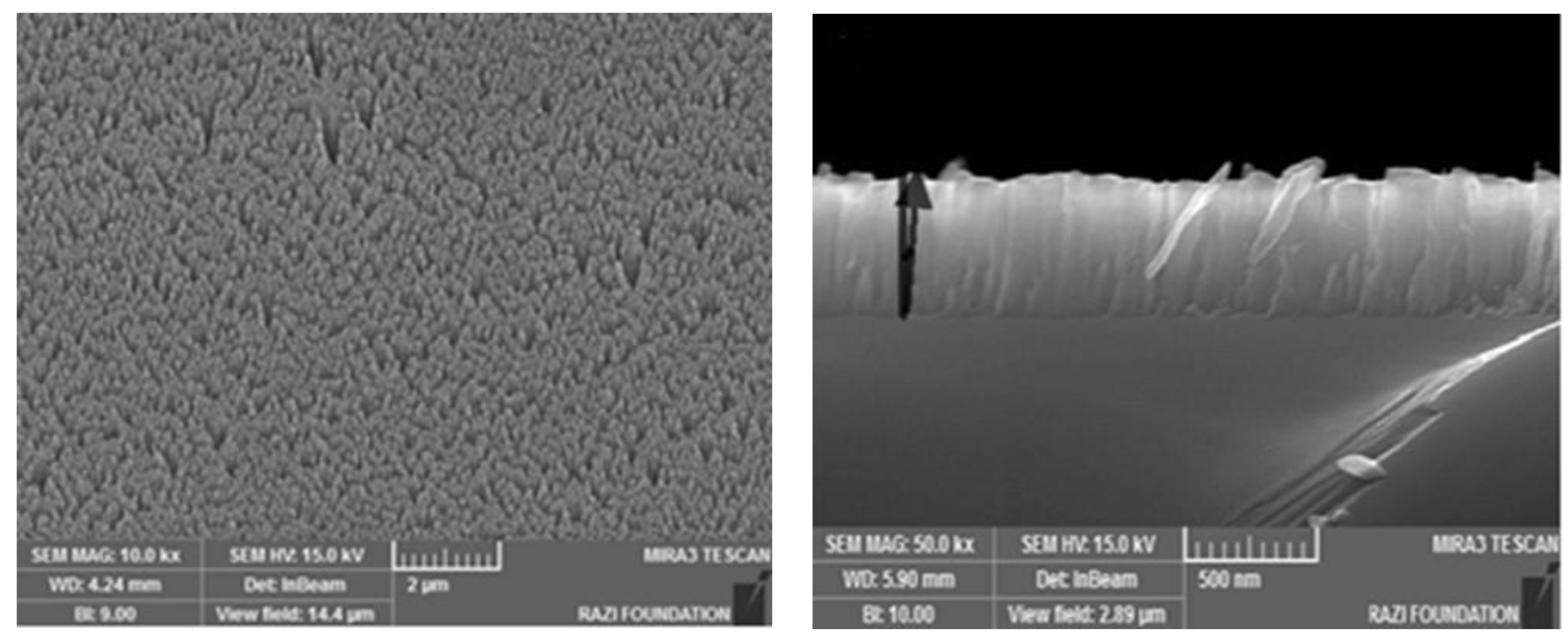

Fig.4.FESEM of electron micrographs of the surface and cross section of $\mathrm{TiO}_{2}$ columnar thin film at $\alpha=0^{\circ}$. 

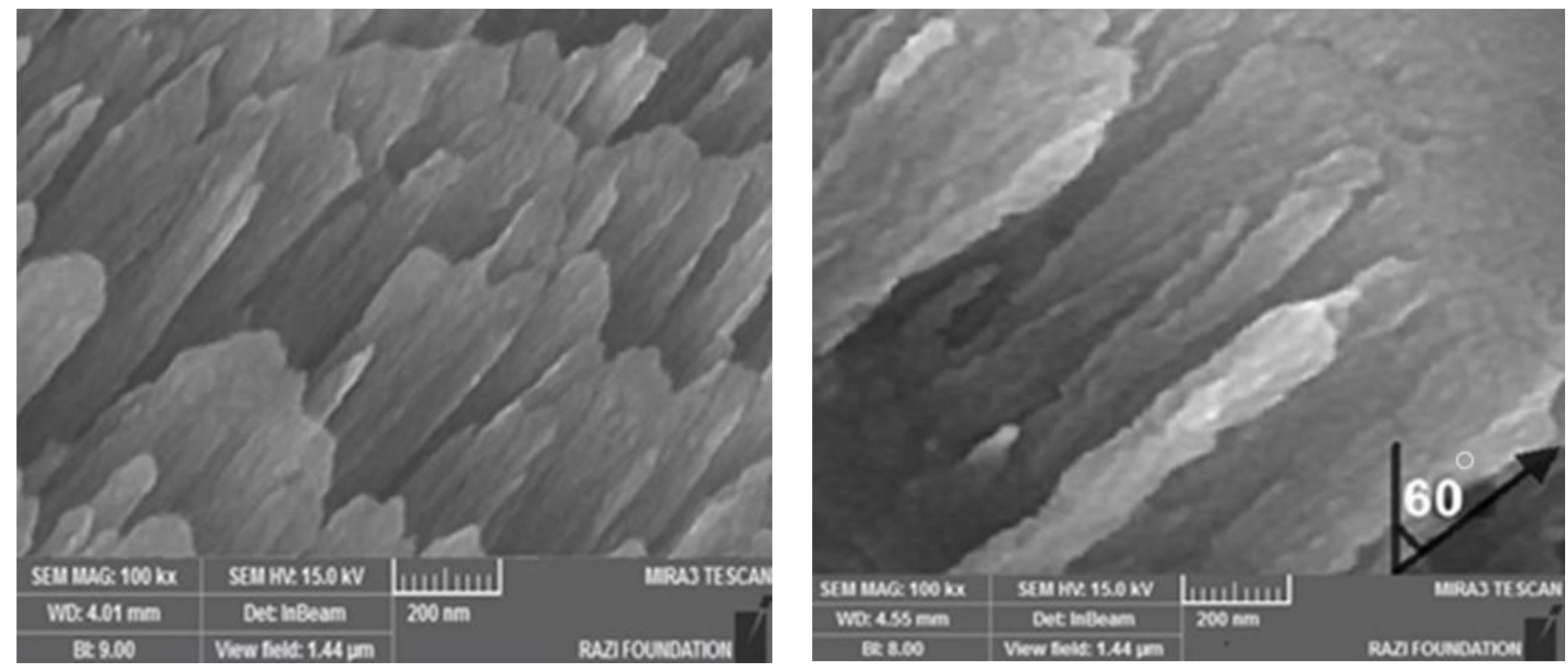

Fig.5.FESEM of electron micrographs of the surface $\mathrm{TiO}_{2}$ columnar thin film at $\alpha=75^{\circ}$.
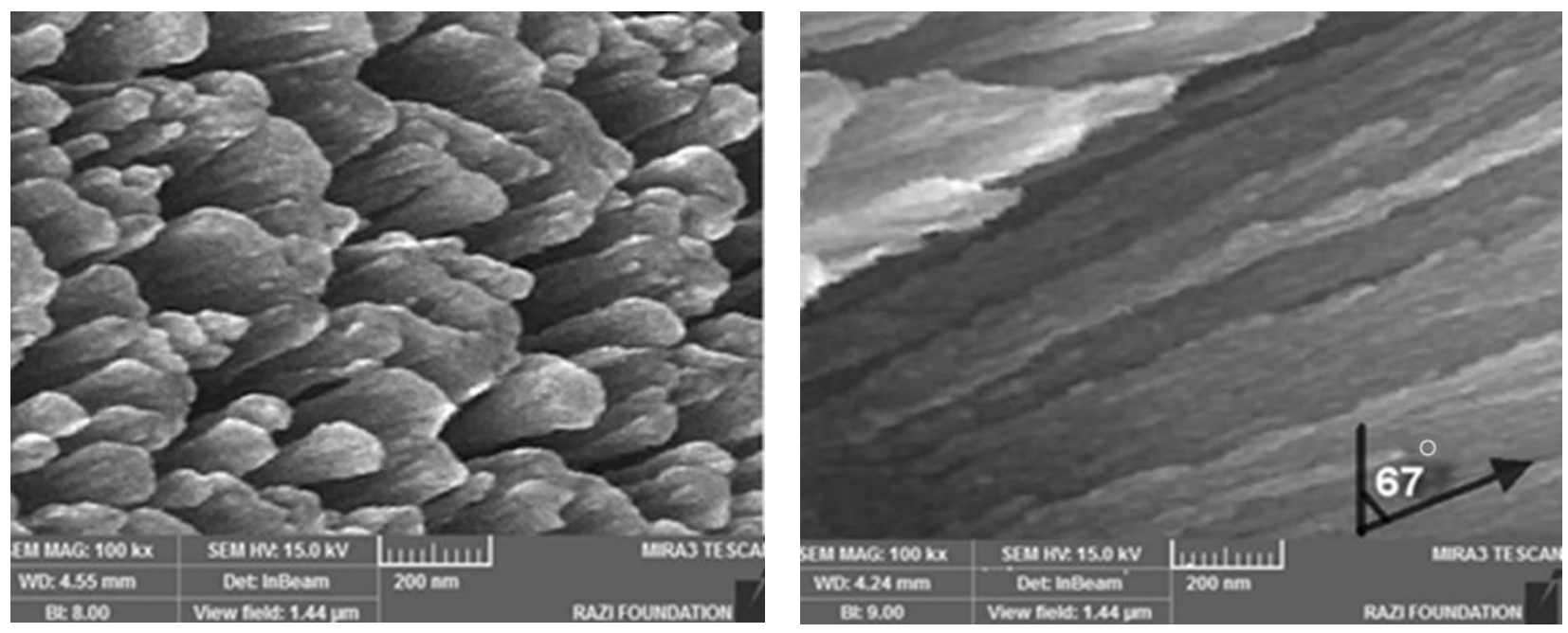

Fig.6.FESEM of electron micrographs of the surface $\mathrm{TiO}_{2}$ columnar thin film at $\alpha=85^{\circ}$.

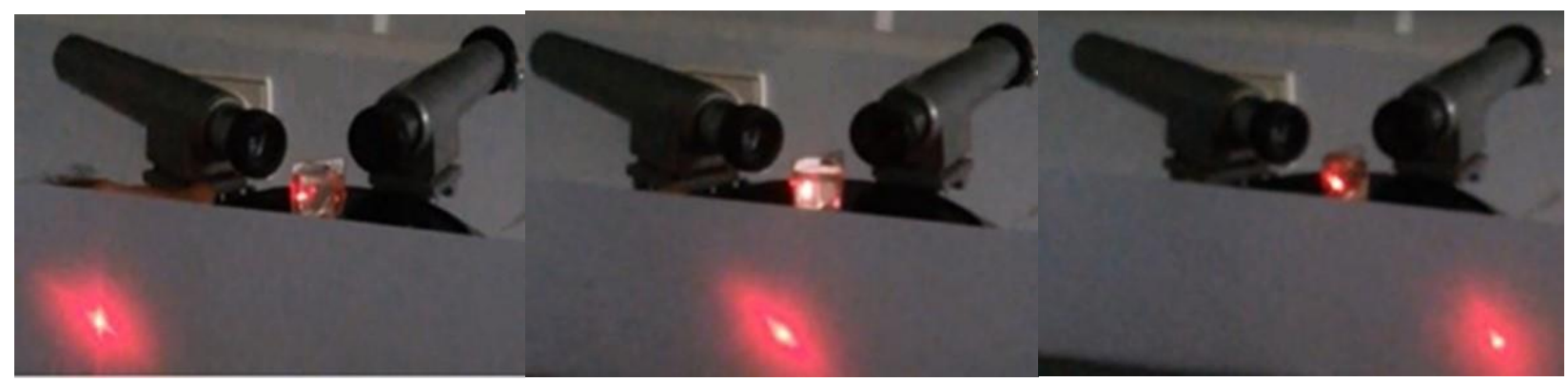

Fig.7. Three different displays of S-polarization light intensity reflected on the screen for silver thin film deposited on microscope slide. The optical calibrated turntable rotates from a low angle $(\theta)$ to more (from left to right). 


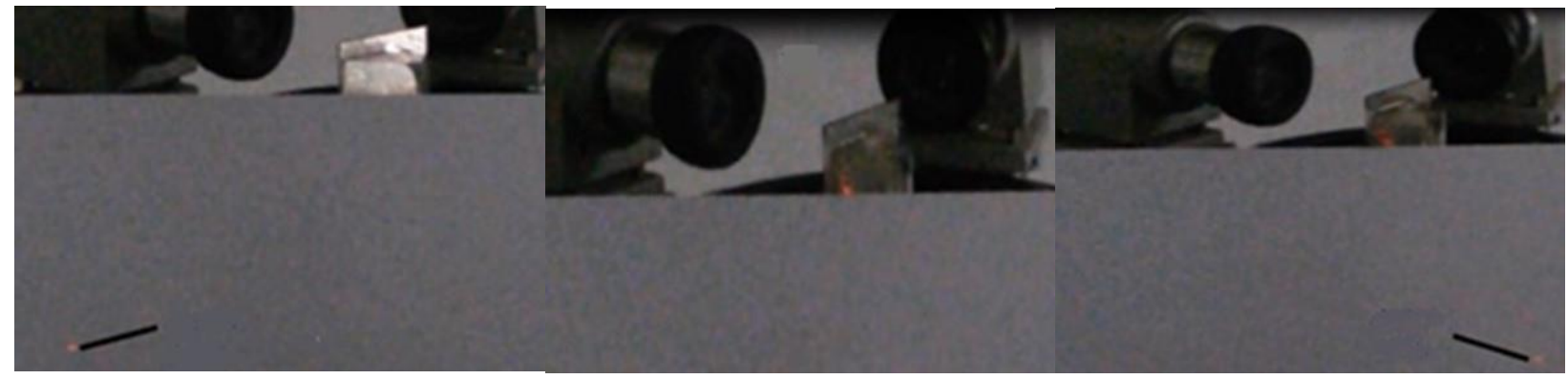

Fig.8. Same as Fig. 7, except that the polarization of light was P.
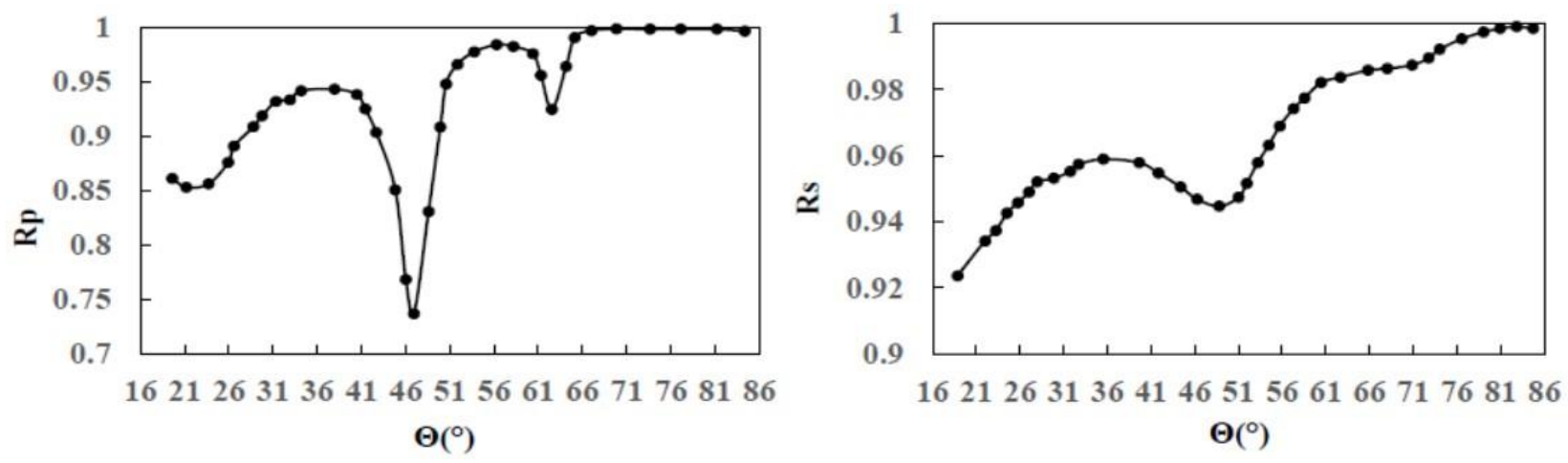

Fig.9.The measured reflection spectra from $\mathrm{TiO}_{2}-\mathrm{Ag}$ composite structure as a function of $\theta$ for both linear polarization at $\alpha=85^{\circ}$.
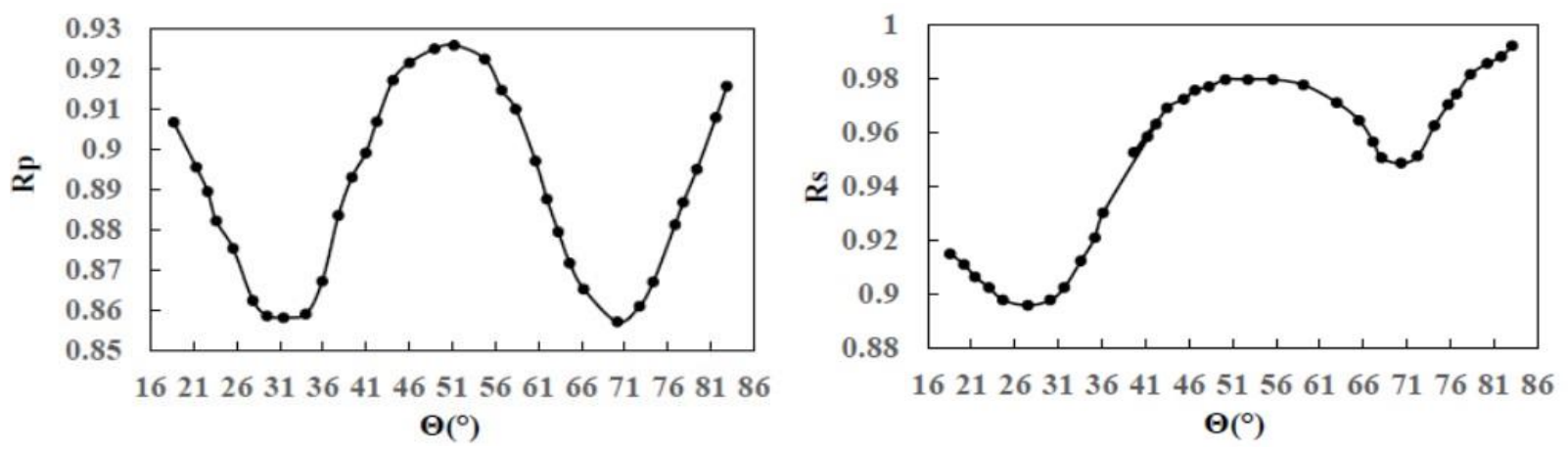

Fig.10. Same as Fig. 9, except that $\alpha=75^{\circ}$.
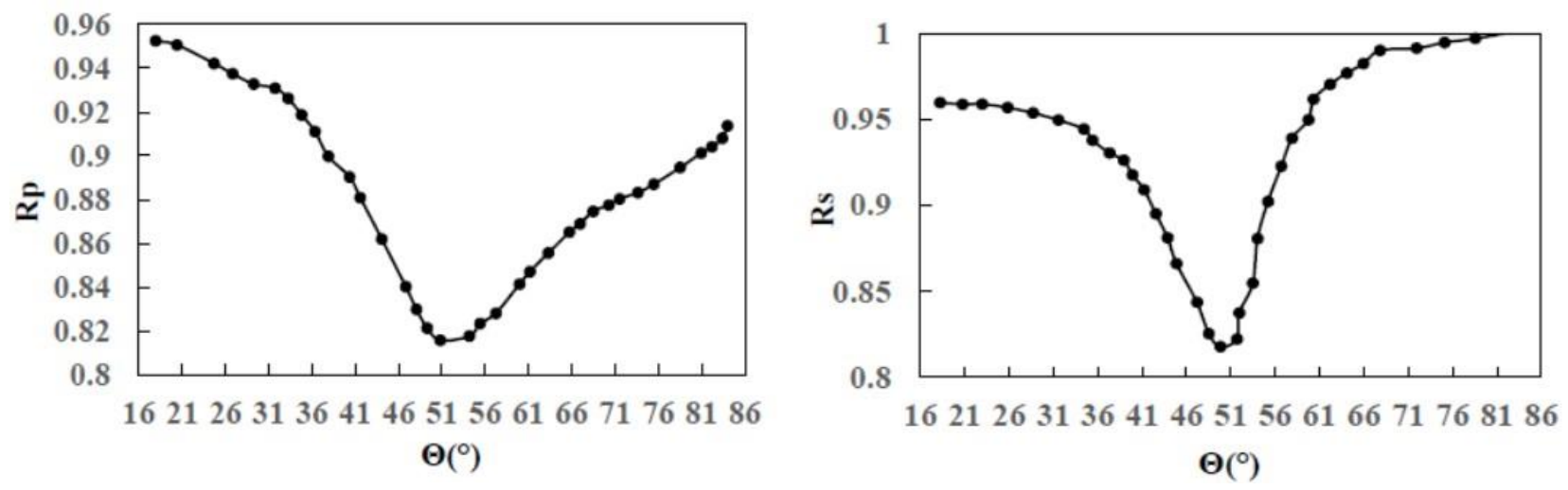

Fig.11. Same as Fig. 9, except that $\alpha=0^{\circ}$. 
Figures

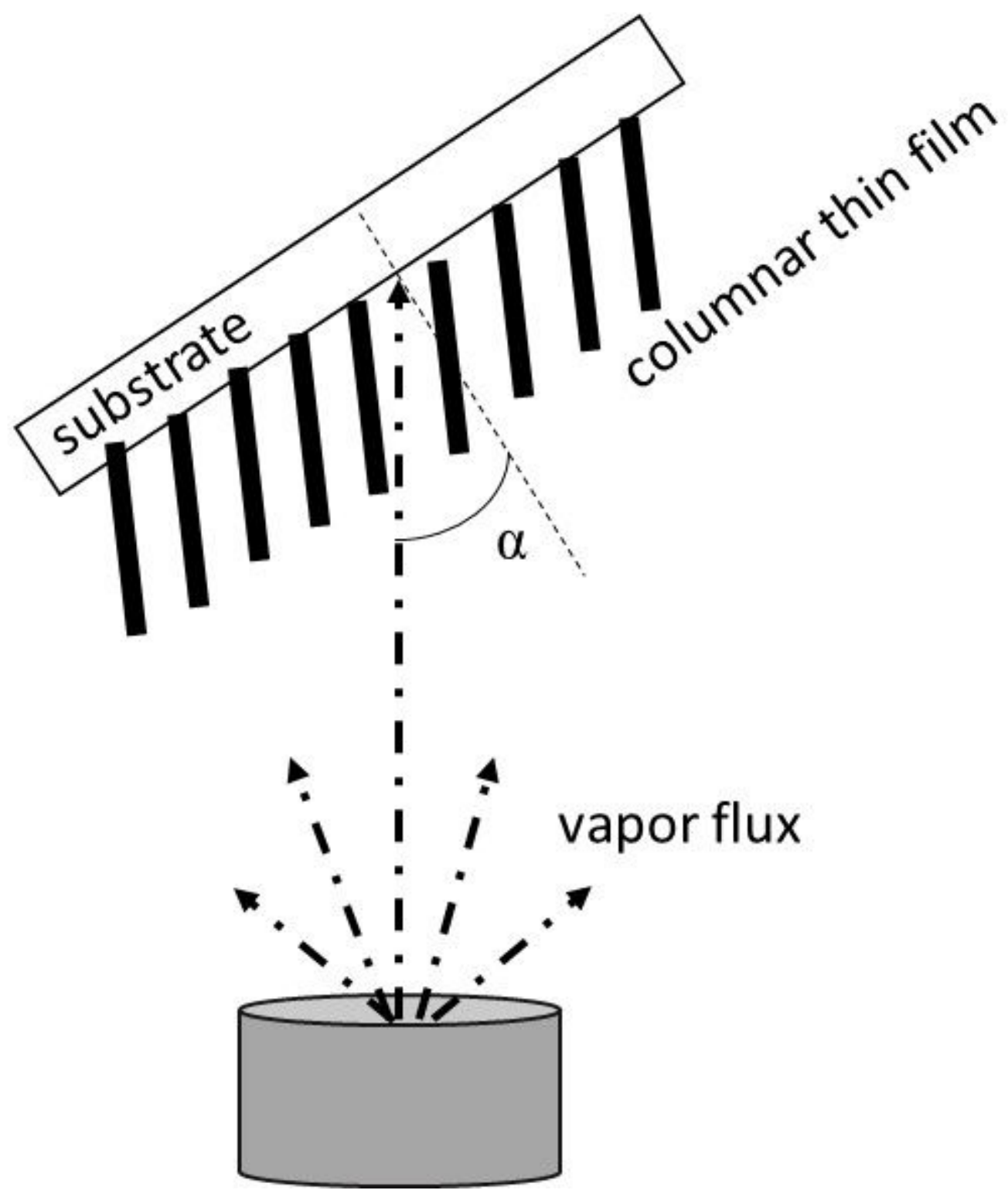

Figure 1

An illustration of oblique angle deposition(OAD) for growth of columnar thin film. The a is deposition angle. 


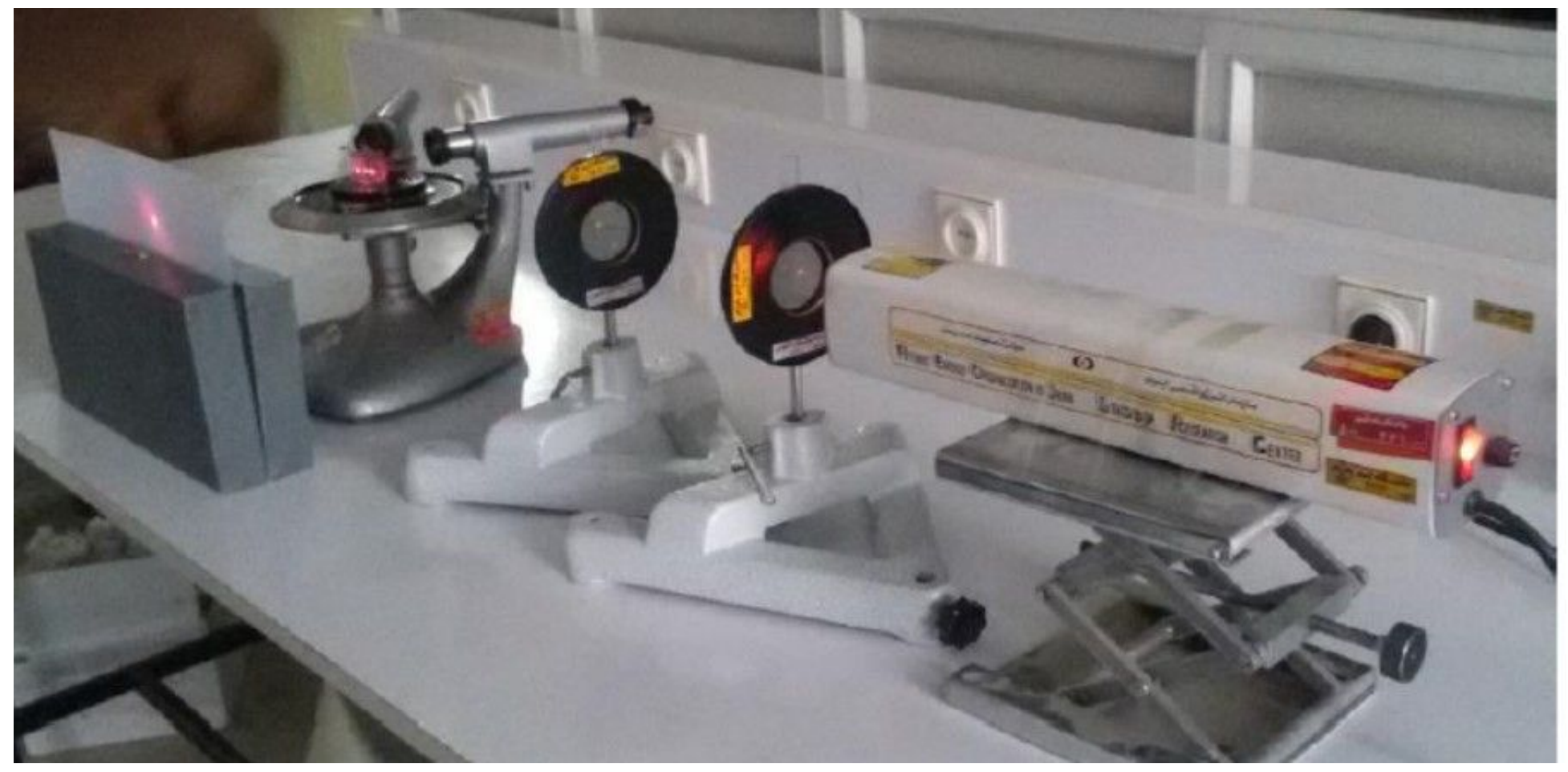

Figure 2

The optical setup for excitation of SPP.

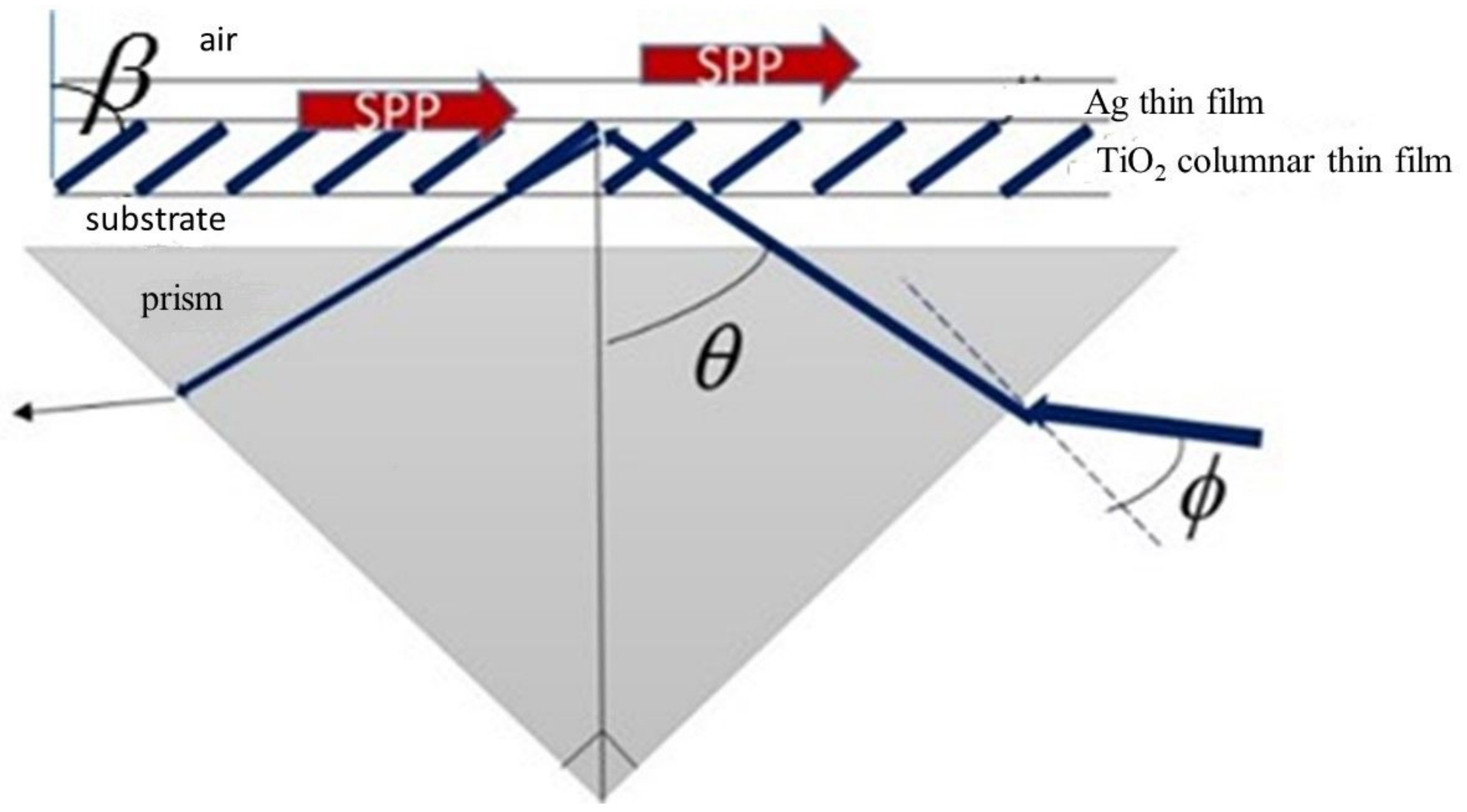

Figure 3

Shematic of two-layer Kretschmann geometry. The $\beta$ is rise angle of columnar thin film. 

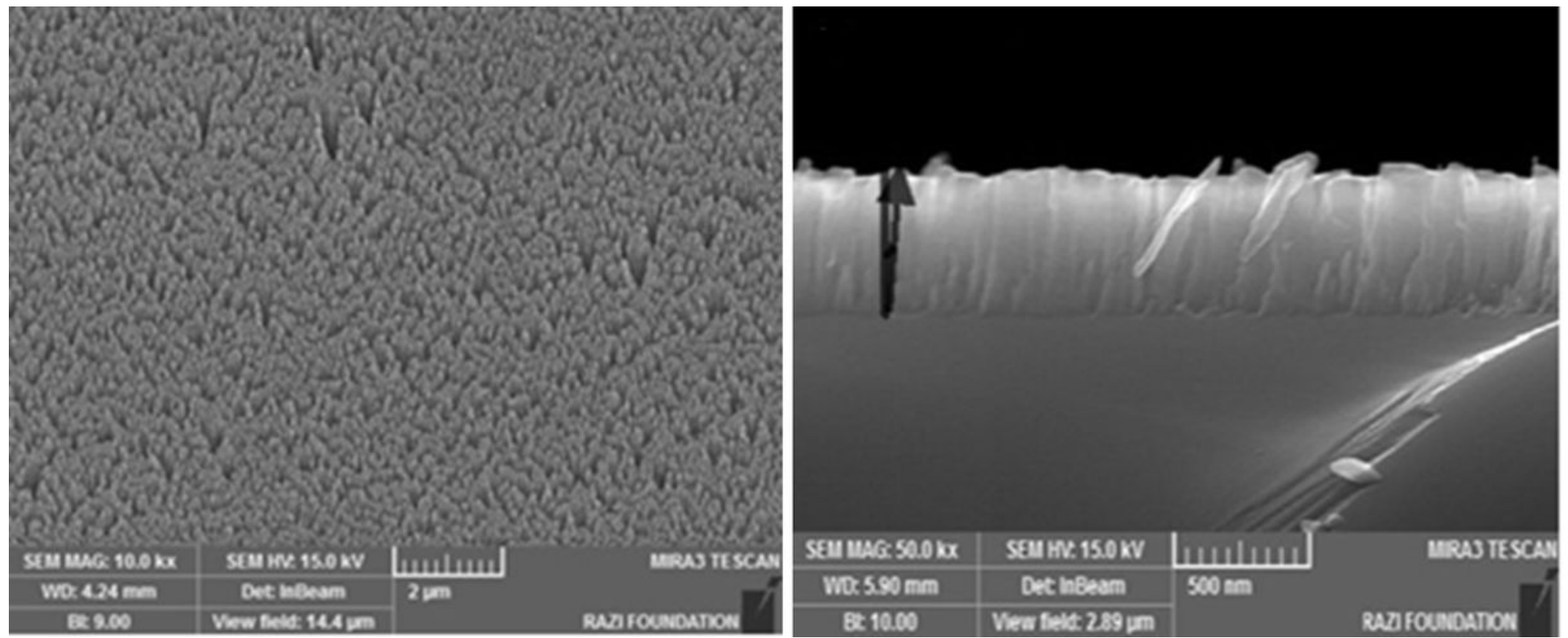

Figure 4

FESEM of electron micrographs of the surface and cross section of TiO2 columnar thin film at $a=0^{\circ}$.
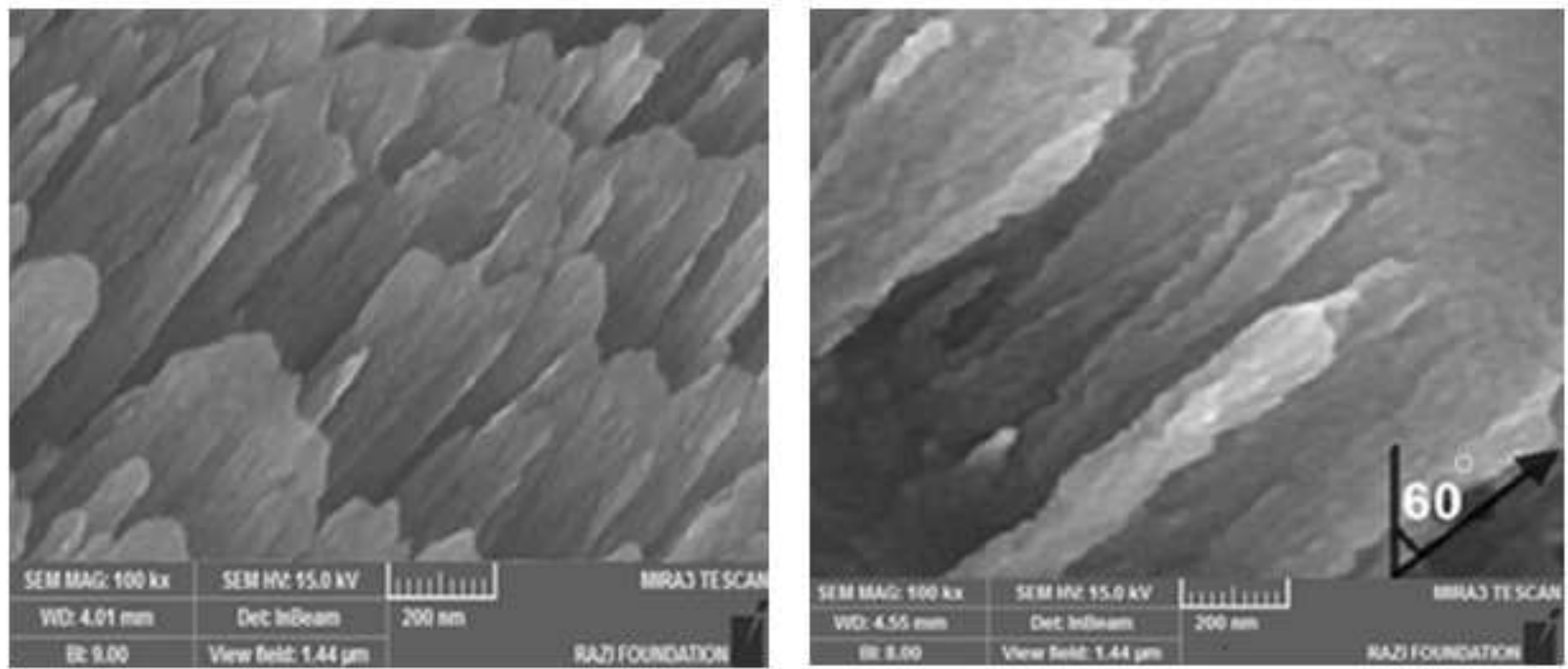

Figure 5

FESEM of electron micrographs of the surface TiO2 columnar thin film at $a=75^{\circ}$. 

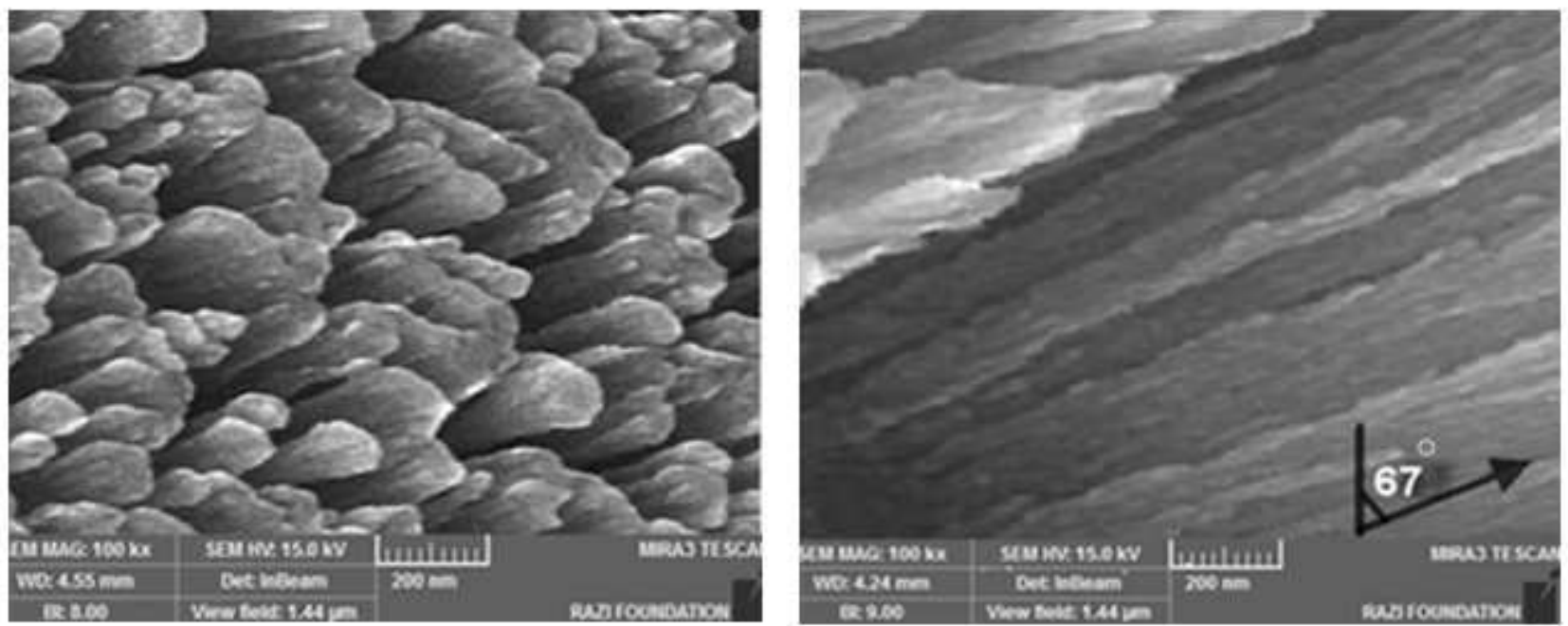

\section{Figure 6}

FESEM of electron micrographs of the surface TiO2 columnar thin film at $a=85^{\circ}$.

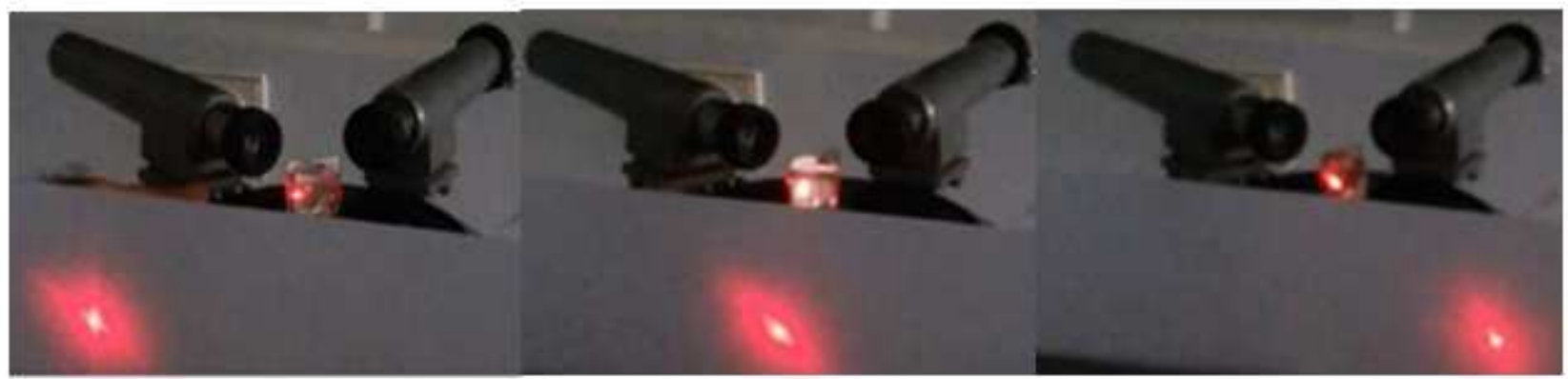

\section{Figure 7}

Three different displays of S-polarization light intensity reflected on the screen for silver thin film deposited on microscope slide. The optical calibrated turntable rotates from a low angle $(\theta)$ to more (from left to right).

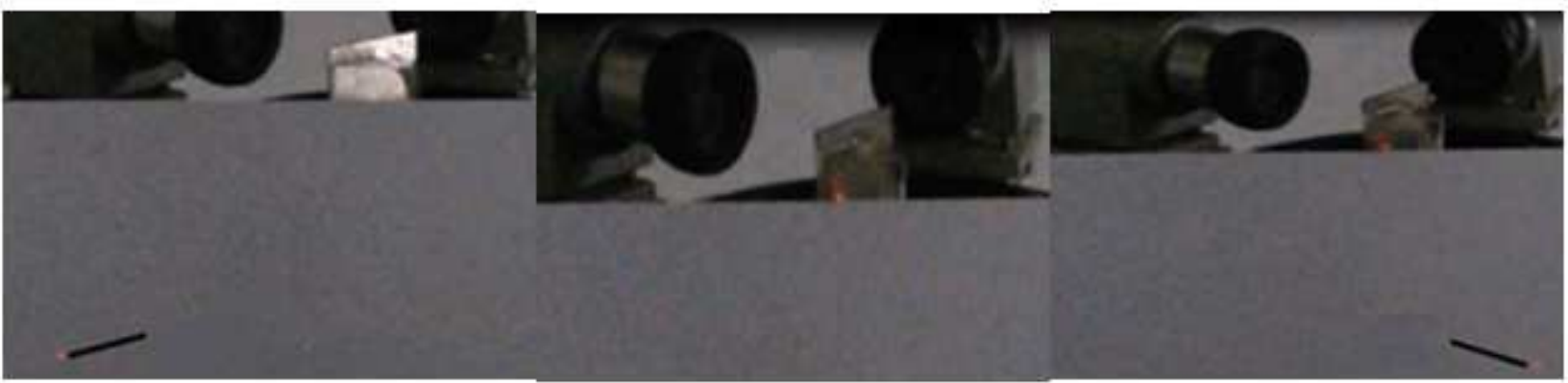

\section{Figure 8}

Same as Fig. 7, except that the polarization of light was P. 

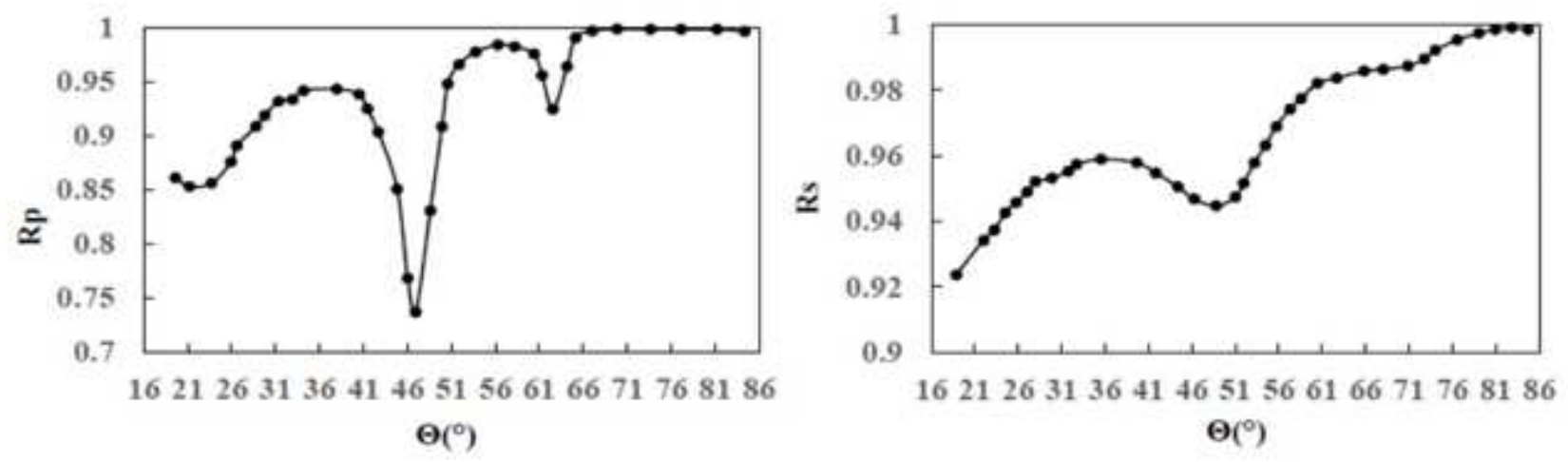

Figure 9

The measured reflection spectra from TiO2 - Ag composite structure as a function of $\theta$ for both linear polarization at $\mathrm{a}=85^{\circ}$.
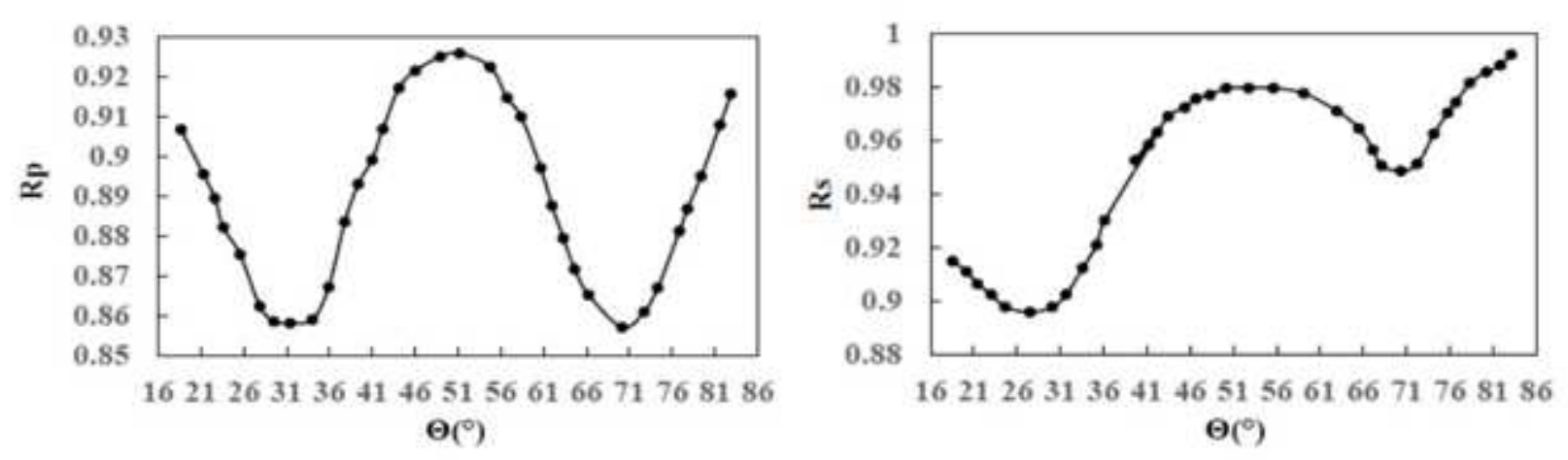

\section{Figure 10}

Same as Fig. 9, except that $a=75^{\circ}$.
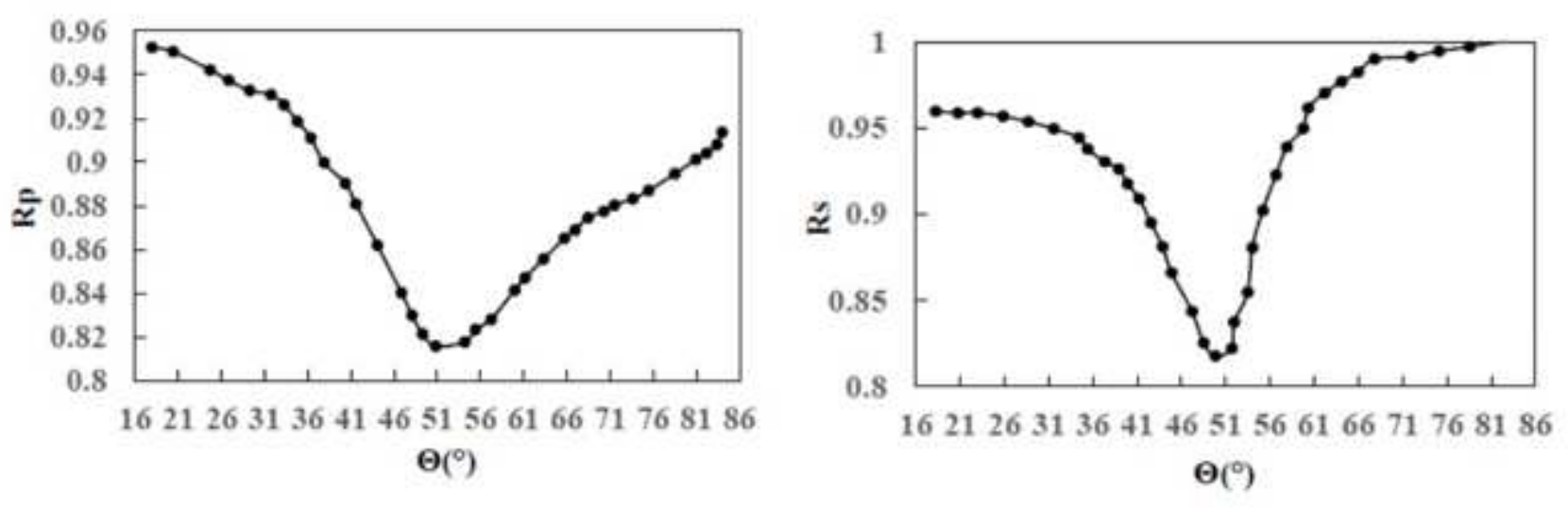

Figure 11

Same as Fig. 9, except that $a=0^{\circ}$. 